\title{
Ectopic Expression of a Cell-Wall-Degrading Enzyme-Induced OsAP2/ ERF 152 Leads to Resistance against Bacterial and Fungal Infection in Arabidopsis
}

\author{
Shakuntala E. Pillai, ${ }^{1}$ Chandan Kumar, ${ }^{2}$ Madhumita Dasgupta, ${ }^{3}$ Bipin K. Kumar, ${ }^{1}$ Sridivya Vungarala, ${ }^{1}$ Hitendra K. Patel, \\ and Ramesh V. Sonti $1,2, \dagger$ \\ ${ }^{1}$ Council of Scientific and Industrial Research (CSIR) Centre for Cellular and Molecular Biology, Hyderabad, Telangana 500007, India \\ 2 National Institute of Plant Genome Research, New Delhi110067, India \\ ${ }^{3}$ Indian Council of Agricultural Research complex for North Eastern Hill Region, Imphal, Manipur795004, India \\ Accepted for publication 30 December 2019.
}

\begin{abstract}
Pathogen secreted cell-wall-degrading enzymes (CWDEs) induce plant innate immune responses. The expression of rice transcription factor APETALA2/ethylene response factor-152 (OsAP2/ERF152) is enhanced in leaves upon treatment with different CWDEs and upon wounding. Ectopic expression of OsAP2/ERF152 in Arabidopsis leads to induction of immune responses such as callose deposition and upregulation of both salicylic acid- and jasmonic acid/ethyleneresponsive defense genes. Arabidopsis transgenics expressing OsAP2/ ERF152 exhibited resistance to infections caused by both bacterial and
\end{abstract}

ABSTRACT

Plant cell-wall-degrading enzymes (CWDEs) are important components of the virulence repertoire of microbial pathogens. However, the damage that they cause results in release of cell wall degradation products which serve as damage-associated molecular patterns (DAMPs) and elicit plant immune responses. Xanthomonas oryzae pv. oryzae, the bacterial pathogen that causes bacterial blight disease in rice, secretes a diverse arsenal of CWDEs such as cellulaseA (ClsA), cellobiosidaseA (CbsA), lipase/esteraseA (LipA), and xylanase (XynB). Prior treatment of rice leaves with CWDEs enhances tolerance to subsequent infection with $X$. oryzae pv. oryzae (Jha et al. 2007). Transcriptome analyses performed in rice leaves indicated that a number of transcription factors are differentially expressed at $12 \mathrm{~h}$ after treatment with either ClsA (26 of 152 differentially expressed genes) or LipA (68 of 720 differentially expressed genes) (Jha et al. 2010; Ranjan et al. 2015). We initiated this study to characterize the APETALA2/ethylene response factor-152 (OsAP2/ERF152) transcription factor because it was highly upregulated in the transcriptome analysis performed upon treatment of rice leaves with ClsA (Jha et al. 2010).

The members of the $A P 2 / E R F$ gene family regulate functions related to development and environmental stress responses in plants (Licausi et al. 2013). Most of the AP2/ERF proteins are expressed in response to the ethylene (ET) hormone (Licausi et al. 2013; Sharoni

†Corresponding author: R. V. Sonti; sonti@ccmb.res.in, rameshvsonti@gmail.com

Funding: This work was supported by grants to R. V. Sonti from the Plant-Microbe and Soil Interactions project of the CSIR, Government of India. This work was also supported by a J. C. Bose fellowship to R. V. Sonti from the Department of Science and Technology, Government of India. S. E. Pillai acknowledges the University Grants Commission, New Delhi, for the Ph.D. fellowship.

*The $e$-Xtra logo stands for "electronic extra" and indicates that four supplementary figures and three supplementary tables are published online.

The author(s) declare no conflict of interest.

(c) 2020 The American Phytopathological Society fungal pathogens (Pseudomonas syringae pv. tomato DC3000 and Rhizoctonia solani AG1-IA, respectively). Ectopic expression of OsAP2/ ERF152 results in transient activation of mitogen-activated protein kinases 3/6 (MPK3/6), which could be leading to the induction of a broad range immunity in Arabidopsis.

Keywords: AP2/ERF transcription factor, callose deposition, cell-walldegrading enzymes, MPK3/6, OsAP2/ERF152, Pseudomonas syringae, Rhizoctonia solani. et al. 2011); however, members of this transcription factor family are also reported to be expressed in response to jasmonic acid (JA) and abscisic acid. Several AP2/ERF transcription factors are known to function as positive or negative regulators of plant immune responses (Licausi et al. 2013). We have identified two AP2/ERF transcription factors, LOC_Os08g36920.1 and LOC_Os01g21120.1, that were upregulated at $12 \mathrm{~h}$ after treatment of rice leaves with either ClsA or LipA enzymes (Supplementary Table S1). Among the two transcription factors, the expression of OsAP2/ERF 152 (LOC_Os08g36920.1) was found to be upregulated to a higher extent. In our previous report, the transient overexpression of OsAP2/ERF152 was found to induce callose deposition and programmed cell death as well as enhance tolerance to subsequent infection with $X$. oryzae pv. oryzae (Jha et al. 2010). In the present study, we wanted to assess the effect of ectopic expression of OsAP2/ERF152 in the induction of immune responses in a heterologous system, Arabidopsis thaliana. Our results demonstrate that the ectopic expression of OsAP2/ERF152 leads to enhanced callose deposition, expression of both the salicylic acid (SA)- and JA/ET-responsive defense genes, and transient activation of MPK3 and -6 in Arabidopsis. This results in resistance to infections caused by a hemibiotroph, Pseudomonas syringae pv. tomato and a necrotroph, Rhizoctonia solani AG1-IA. Our results suggest that ectopic overexpression of OsAP2/ERF152 can be a way of enhancing tolerance to hemibiotrophic and necrotrophic pathogens in plants.

\section{MATERIALS AND METHODS}

Plant materials and growth conditions. All of the experiments in rice were performed in bacterial leaf-blightsusceptible rice cultivar Taichung Native-1 (TN-1) grown in the greenhouse. OsAP2/ERF152 transgenic lines were generated in A. thaliana Columbia ecotype (Col-0). For all of the experiments, Arabidopsis seed were sterilized and germinated on $0.5 \times$ Murashige and Skoog (MS) agar medium (Murashige and Skoog 1962). Seven-day-old 
Arabidopsis seedlings were transferred to soil (vermiculite/perlite/tissue culture mix, 1:1:2 ratio) and grown in a plant growth chamber maintained at $22^{\circ} \mathrm{C}$ with a 12-h day-and-night cycle.

CWDEs and elicitor treatment in rice leaves. The expression levels of $O s A P 2 / E R F 152$ gene transcript were studied in rice upon treatment with various CWDEs and elicitors, as described earlier (Pillai et al. 2018). Briefly, leaves ( $n=15$ to 20) of 2-week-old TN-1 rice seedlings were infiltrated with one of the following using a needleless syringe: xylanase $(2 \mathrm{U} / \mathrm{ml})$, pectinase (2 U/ml), flagellin $22(\mathrm{Flg} 22 ; 100 \mu \mathrm{M})$, lipopolysaccharide (LPS; $100 \mu \mathrm{M}$ ), or sterile water. For studying response to wounding, leaves of the rice seedlings were pin pricked 8 to 10 times and sprayed with water. Leaves were collected after $12 \mathrm{~h}$ of the treatment and further processed for RNA extraction and quantitative PCR (qPCR) analysis for studying the expression levels of OsAP2/ERF152. Each experiment had three biological replicates and three such independent experiments were performed.

Localization studies in onion epidermis. For localization studies, the OsAP2/ERF152 gene was cloned (excluding stop codon) into the $\mathrm{pH} 7 \mathrm{FWG} 2$ plant expression vector using Gateway cloning. The gene was expressed constitutively under the CaMV $35 \mathrm{~S}$ promoter and with a green fluorescent protein (GFP) tag at the C-terminal region of the protein. For localization studies, Agrobacterium strain AGL1 carrying pH7FWG2::OsAP2/ERF152 was syringe infiltrated into onion epidermal cells (Xu et al. 2014). Briefly, an overnight-grown culture of Agrobacterium was diluted to an optical density at $600 \mathrm{~nm}\left(\mathrm{OD}_{600}\right)$ of 0.01 in infiltration buffer (42 mM dextrose, $100 \mathrm{mM} \mathrm{CaCl}_{2}, 0.01 \mathrm{mM}$ 6-benzylaminopurine [BAP], 0.01\% Silwet L-77, $0.05 \mathrm{mM} \mathrm{MgCl}_{2}, 12.5 \mathrm{mM}$ acetosyringone, and $100 \mathrm{mM}$ morpholineethanesulfonic acid [pH 5.6] solution). For transformation, 100 to $200 \mu$ lof the above suspension was injected into the onion scales between adaxial epidermis and mesophyll tissue using a needled syringe. The onion bulb was incubated at $28^{\circ} \mathrm{C}$ for $48 \mathrm{~h}$. The epidermis was peeled off and placed on a slide over a drop of mounting medium containing $4^{\prime}, 6-$ diamidino-2-phenylindole (DAPI) (Fluorshield; Calbiochem). The samples were imaged under GFP, DAPI, and phase contrast filters using an ECLIPSE Ni-E epifluorescence microscope (Nikon).

Microbial growth conditions. $P$. syringae pv. tomato DC3000 culture was grown on King's basal agar medium (HiMedia). For all assays, Agrobacterium strains and $P$. syringae pv. tomato were grown in Luria-Bertani (LB) broth (HiMedia) at $28^{\circ} \mathrm{C}$ for $18 \mathrm{~h}$. The fungal pathogen $R$. solani AG1-IA was grown and maintained on potato dextrose agar medium (HiMedia) at $28^{\circ} \mathrm{C}$.

Construction of plant expression vector. The cDNA was synthesized from the total RNA isolated from ClsA enzyme-treated rice leaves (12 h posttreatment) using Superscript III reverse transcription (Invitrogen). The OsAP2/ERF152 (LOC_Os08g36920.1) gene without the stop codon was cloned into the $17-\beta$-estradiolinducible plant-expression vector pMDC7 using Gateway cloning as per the manufacturer's instructions. The pMDC7::OsAP2/ERF 152 vector was transformed into Agrobacterium tumefaciens strain LBA4404 by electroporation and the transformed clones were selected on a medium containing the appropriate antibiotics (Supplementary Table S2). The LBA4404/pMDC7::OsAP2/ERF152 clones were confirmed for the construct by colony PCR and subsequent sequencing of the PCR amplicons by using pMDC7specific primers (Supplementary Table S3).

Generation of stable transgenic Arabidopsis plants. Arabidopsis Col-0 plants (wild type) were transformed with A. tumefaciens LBA4404 carrying pMDC7::OsAP2/ERF152 by the floral dip method (Clough and Bent 1998). The transformed seedlings were selected on MS agar with hygromycin $(25 \mu \mathrm{g} / \mathrm{ml})$. The presence of the OsAP2/ERF152 gene in the putative $\mathrm{T}_{1}$ transgenic plants was assessed by genotyping using direct PCR (Terra PCR direct polymerase kit; Clontech) with T-DNA-specific primers (Supplementary Table S3) followed by sequencing of the amplified product. All functional studies were performed on $\mathrm{T}_{2}$ progenies of two independent transgenic lines (numbers XVE:: OSAP2/ERF152-5 and -15), in which high levels of OSAP2/ERF 152 expression were observed after estradiol treatment.

Callose deposition assay. Wild-type and transgenic Arabidopsis plants that were at the rosette stage ( 4 weeks old) were used for callose deposition assays. The leaves were infiltrated either with estradiol $(20 \mu \mathrm{M})$, which is the inducer of the XVE promoter, or with water using a 1-ml needleless syringe. At $12 \mathrm{~h}$ postinfiltration, the leaves were harvested and processed for callose staining by fixing in ethanol/acetic acid (3:1) solution to remove the chlorophyll completely. The leaves were then rehydrated by $2 \mathrm{~h}$ of $70 \%$ ethanol treatment, followed by $2 \mathrm{~h}$ of water treatment. The callose deposits were stained by soaking the leaves for 16 to $18 \mathrm{~h}$ in $0.01 \%$ (wt/vol) aniline blue dye dissolved in $150 \mathrm{mM}$ potassium-phosphate buffer (pH 9.5) (Millet et al. 2010). The leaves were observed for callose deposits under $10 \times$ magnification of an epifluorescence microscope (ECLIPSE Ni-E; Nikon) using the blue filter (excitation wavelength $=365 \mathrm{~nm}$ and emission wavelength $>420 \mathrm{~nm}$ ). The number of callose deposits per field of view for a minimum of five leaves with three different fields was counted. Three such independent experiments were performed in two independent lines.

Quantitative real-time PCR analysis. RNA was extracted from either rice or Arabidopsis tissues using TRIzol reagent (Thermo Fisher Scientific). The total RNA sample was treated with DNaseI (NEB). Total RNA (1 $\mu \mathrm{g})$ was used for cDNA synthesis using cDNA EcoDry premix kit-Oligo dT (Clontech, Takara). The diluted cDNA sample (1:10 fold) was used for qPCR analysis using Power SYBR green/ROX Master Mix (Thermo Fisher Scientific) on the 7900 HT sequence detection system (Applied Biosystem). For rice and Arabidopsis samples, OsGAPDH and AtUBQ5 genes, respectively, were used as internal controls. All of the primers used for qPCR (Supplementary Table S3) in this study were designed using Quantprime (Arvidsson et al. 2008). The fold change in expression for various genes between water-treated (control) and estradiol-treated samples was calculated using the $2^{-\Delta \Delta C t}$ method (Livak and Schmittgen 2001).

$P$. syringae pv. tomato infection assay in Arabidopsis. The $P$. syringae pv. tomato infection assay was performed in 4-week-old transgenic Arabidopsis (XVE::OsAP2/ERF152) plants. In order to induce $O s A P 2 / E R F 152$ expression, the pots were treated with either only water or water containing $40 \mu \mathrm{M}$ estradiol $24 \mathrm{~h}$ prior to infection. For bacterial infection, the $P$. syringae pv. tomato culture was grown for 16 to $18 \mathrm{~h}$ in LB broth containing rifampicin $(50 \mathrm{mg} /$ liter). The culture was pelleted, washed, and resuspended in sterile $10 \mathrm{mM} \mathrm{MgCl} 2$ solution with an $\mathrm{OD}_{600}$ of 0.01 . The bacterial cell suspension was infiltrated into leaves of pretreated plants. Leaf samples were collected on the 0th and 2nd day postinfiltration for measuring the bacterial growth (Starkey and Rahme 2009). Briefly, the leaves were crushed in sterile $10 \mathrm{mM} \mathrm{MgCl}_{2}$ solution and serially diluted to obtain countable bacterial colonies. The same procedure was repeated with five different plants in two independent lines and the experiments were replicated thrice.

$R$. solani AG1-IA infection assay in Arabidopsis. $R$. solani infection assay was performed on 4-week-old rosette-stage transgenic Arabidopsis plants (XVE::OsAP2/ERF152) by inducing the expression of OsAP2/ERF152 as mentioned above. For $R$. solani infection, the sclerotium was germinated by growing in liquid potato dextrose broth for $24 \mathrm{~h}$ at $28^{\circ} \mathrm{C}$. The fungal culture was resuspended in sterile water by pipetting thoroughly. The induced and uninduced transgenic plants were infected by adding $200 \mu \mathrm{l}$ of the culture suspension at the center of the rosette. The infected plants were monitored for disease symptoms and photographed until the 10th day postinfection. For the estimation of fungal growth in the infected plants, the entire plant was uprooted and frozen in liquid nitrogen. Genomic DNA was isolated from the plants using a plant DNA isolation kit (Sigma). The DNA concentration was measured spectrophotometrically and different dilutions of the total DNA (0.1 to $100 \mathrm{ng}$ ) were subjected to fungal DNA 
quantification. The level of fungal $18 S$ rRNA gene was quantitated in three independent samples by qPCR using $R$. solani-specific primers (Okubara et al. 2008). Normalization was done using the Arabidopsis ubiquitin 5 (AtUBQ5) gene.

Seedling growth inhibition assay. Seven-day-old seedlings $(n=15)$ of either XVE::OsAP2/ERF152 transgenic lines or wildtype Arabidopsis plants were germinated on MS agar and grown vertically on the agar surface in either the presence or absence $20 \mu \mathrm{M}$ estradiol. The fresh weight of each seedling was measured on the 10th day. The experiment was repeated thrice with two independent transgenic lines.

Western blot analysis. For mitogen-activated protein kinase (MAPK) assays, 2-week-old wild-type and transgenic Arabidopsis seedlings ( $n=10$ to 15$)$ were submerged in sterile water with or without $20 \mu \mathrm{M}$ estradiol. The samples were collected after $0,10,15$, 30 , and 60 min of estradiol treatment and frozen in liquid nitrogen. The crude protein was extracted using protein extraction buffer (50 mM Tris- $\mathrm{HCl}$ [pH 7.5], $150 \mathrm{mM} \mathrm{NaCl}, 250 \mathrm{mM}$ mannitol, $5 \mathrm{mM}$ EDTA, $10 \%$ glycerol, $1 \mathrm{mM}$ dithiothreitol, $1 \%$ Triton X100, $1 \mathrm{mM}$ phenylmethylsulfonyl fluoride, and $1 \mathrm{mM} \mathrm{NaF}$ ) along with a plant protease inhibitor cocktail (Sigma-Aldrich). The protein concentration was estimated spectrophotometrically and an equal amount of protein was resolved on $10 \%$ polyacrylamide gel. The MPK6 and -3 proteins were probed using antiphospho p44/42 MAPK (Thr202/ Tyr204) antibody (1:2000; catalog number CST9101S; Cell Signaling Technology). Antirabbit peroxidase conjugated secondary antibody (1:50000; AbCAM) was probed and the protein band was viewed using Luminata Forte HRP substrate (Millipore). The signal was captured using a Vilber Lourmat chemiluminescence imaging system.

\section{RESULTS}

Expression of $O s A P 2 / E R F 152$ is induced upon cell wall damage. The previous microarray analyses of rice leaves indicated that the expression of $O S A P 2 / E R F 152$ was induced by 2 to fourfold at $12 \mathrm{~h}$ posttreatment with either cellulase (ClsA) or esterase (LipA) enzymes (Jha et al. 2010; Ranjan et al. 2015). In order to determine whether OsAP2/ERF152 expression is induced with other kinds of CWDEs, we treated rice leaves with commercially available pectinase and xylanase. The expression of OsAP2/ERF152 was induced four- and twofold after treatment with pectinase and xylanase, respectively (Fig. 1). Wounding of rice leaves also induced the expression of OsAP2/ERF152. However, treatment

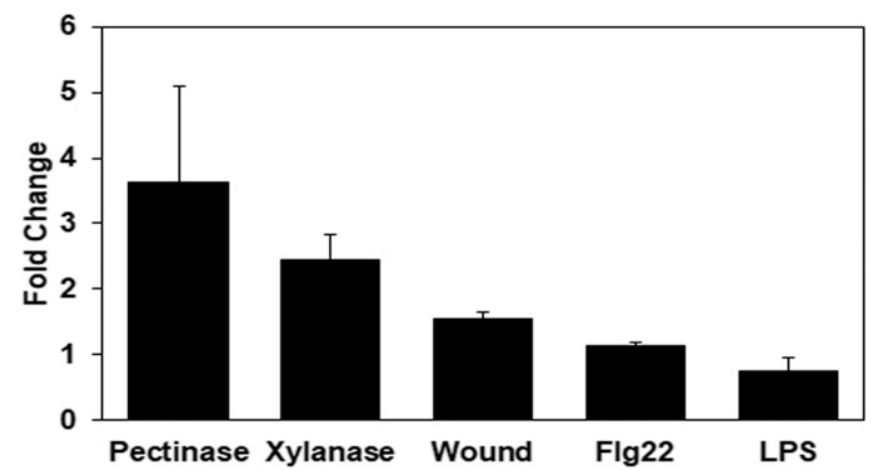

Fig. 1. Expression of $O s A P 2 / E R F 152$ is induced upon treatment with cellwall-degrading enzymes. Young leaves of 2 -week-old Taichung Native- 1 rice seedlings ( $n=15$ to 20 ) were infiltrated with one of the following: control (water), pectinase $(2 \mathrm{U} / \mathrm{ml})$, xylanase $(2 \mathrm{U} / \mathrm{ml})$, flagellin $22(\mathrm{Flg} 22 ; 100 \mu \mathrm{M})$, or lipopolysaccharide (LPS; $100 \mu \mathrm{M})$. Leaves $(n=15)$ were also injured 10 times using a 1-ml syringe needle for wound treatment. At $12 \mathrm{~h}$ after the treatment, leaves were harvested and processed for quantitative PCR. The relative fold change was calculated over water-treated or untreated (for wound treatment) samples. $O s G A P D H$ was used as internal control. The graph represents the average from three biological replicates and the error bar denotes standard deviation. with pathogen-associated molecular patterns (PAMPs) such as Flg22 and LPS failed to induce OsAP2/ERF152 expression in rice. OSAP2/ERF152 transcription factor has a nuclear localization sequence (from 132 to 140 amino acids) (Jha et al. 2010); hence, we studied its localization in plant cells. GFP-tagged OsAP2/ERF152 was transiently expressed in onion epidermal cells for localization studies. OsAP2/ERF152-GFP merges with DAPI-stained nucleus and confirms localization into the nucleus (Supplementary Fig. S1).

Ectopic expression of OsAP2/ERF 152 leads to the induction of callose deposition in Arabidopsis. Jha et al. (2010) reported that the transient overexpression of OsAP2/ $E R F 152$ led to enhanced callose deposition in rice seedlings. In order to determine whether ectopic expression of OsAP2/ERF152 induces callose deposition in Arabidopsis, we generated stably transformed transgenic lines expressing OsAP2/ERF152 under an estradiol-inducible promoter (XVE::OsAP2/ERF152). The $\mathrm{T}_{2}$ progeny of two independently transformed XVE::OsAP2/ERF 152 transgenic lines that expressed higher levels of OsAP2/ERF152 (3to 10-fold) in a qPCR analysis were used for all studies (Supplementary Fig. S2). Leaves of the wild type and XVE:: OsAP2/ERF 152 were infiltrated with either estradiol or water. Leaves were collected after $12 \mathrm{~h}$ and stained for callose deposition. Ectopic expression of OsAP2/ERF152 was found to induce callose deposition (approximately fivefold higher) as compared with the control (water-treated) leaves (Fig. 2A and B). Similar results were obtained in three different experiments performed in two independently transformed transgenic lines. Treatment with estradiol alone did not induce callose deposition in the wild-type Arabidopsis plants (Kachewar et al. 2019; Pillai et al. 2018) (Fig. 2B).

Ectopic expression of OsAP2/ERF152 resulted in enhanced expression of SA- and JA/ET-responsive defense genes in Arabidopsis. Plant immune responses are controlled by plant defense hormones such as SA, JA, and ET. These hormones regulate the expression of many defense response genes which actually act to inhibit growth of the invading pathogens. The expression of defense response genes regulated by different phytohormones was analyzed in XVE::OsAP2/ERF152 plants in the presence or absence of estradiol. Upon induction of $O s A P 2 /$ ERF152 expression, the transcript levels of Pathogenesis related-1 (PR1), PR2, PR3, Phytoalexin deficient-3(PAD3), and avrPphB susceptible-3 $(P B S 3)$ were significantly upregulated when the average cycle threshold $(\mathrm{Ct})$ values were compared with that of the uninduced samples using Student's $t$ test $(P<0.05)$ (Fig. 2C). Both SA-responsive $(P R 1, P R 2, P A D 3$, and $P B S 3)$ as well as JAresponsive $(P R 3)$ defense genes were upregulated on induction of expression of OsAP2/ERF152 in the Arabidopsis transgenic lines. We further tested the expression of other biosynthetic and responsive genes whose levels are regulated by either SA or JA/ ET. Interestingly, OsAP2/ERF152 expression induced the expression of genes that are regulated by the SA hormone (Isochorismate synthase and SA glycosyl transferase), JA hormone (Allene oxide synthase-1, coronatine-insensitive protein-1, and Jasmonate zim domain), or ET hormone (Ethylene response factor 1 [ERF1], ethylene insensitive-2, and 1-aminoacyclopropane 1-carboxylate synthase) (Fig. 2D). We found that the expression of OsAP2/ ERF152 results in the upregulation of SA- as well as JA/ETresponsive genes in Arabidopsis. Treatment with estradiol alone did not induce expression of either SA- or JA/ET-responsive genes in the wild-type Arabidopsis plants (Kachewar et al. 2019) (Supplementary Fig. S3).

Expression of $O s A P 2 / E R F 152$ provides resistance to $P$. syringae infection in Arabidopsis. In a previous study, transient overexpression of OsAP2/ERF152 was found to lead to resistance against subsequent $X$. oryzae pv. oryzae infection in rice (Jha et al. 2010). In order to determine whether ectopic expression of $O s A P 2 / E R F 152$ would enhance tolerance to bacterial infection in another plant, we inoculated estradiol-inducible XVE::OsAP2/ ERF152 transgenic lines with $P$. syringae pv. tomato DC3000 in the 
presence and absence of estradiol. Induction of OsAP2/ERF152 expression resulted in a two- to threefold reduction in the total average bacterial count (Fig. 3A) as compared with the uninduced condition. Thus, ectopic expression of OsAP2/ERF152 in Arabidopsis led to resistance to $P$ syringae pv. tomato infection, which is a hemibiotrophic pathogen. Treatment with estradiol alone did not affect $P$. syringae pv. tomato disease progression in the wild-type Arabidopsis plants (Kachewar et al. 2019).
A

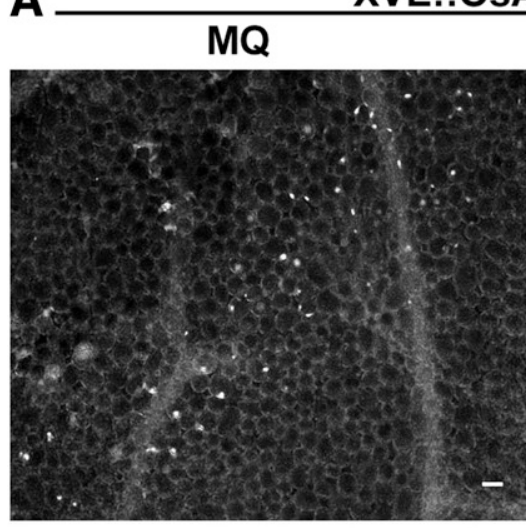

XVE::OSAP2/ERF152

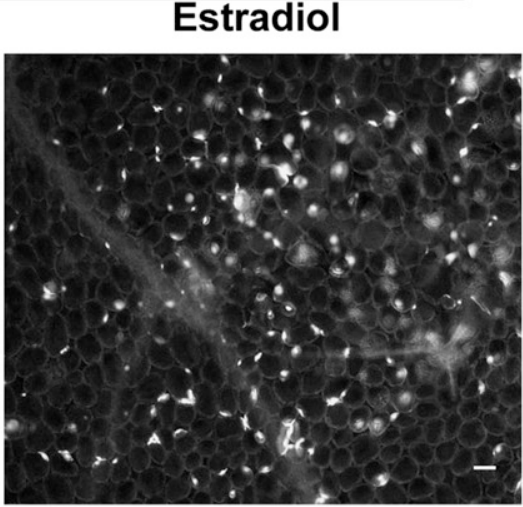

B

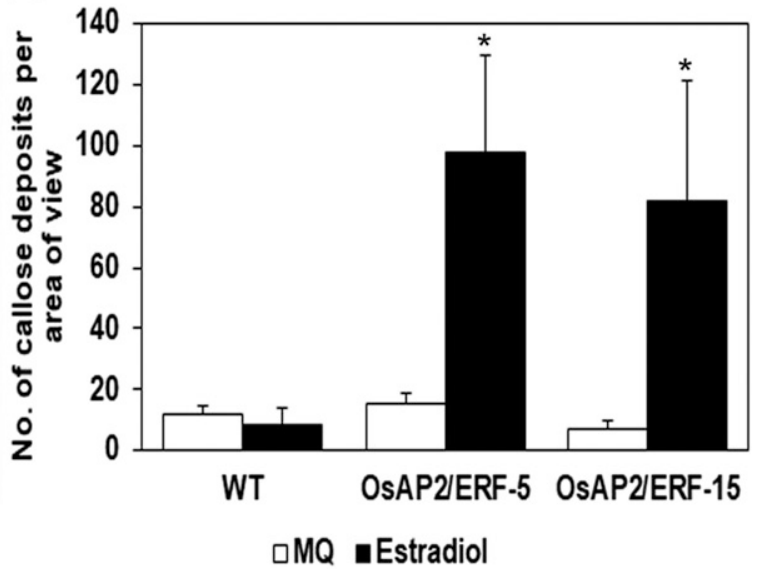

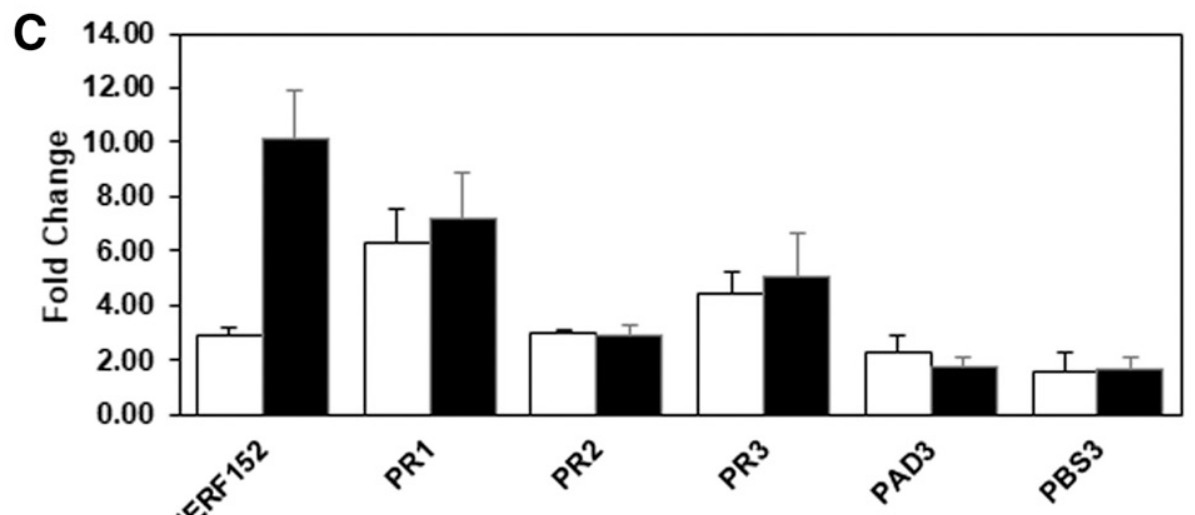

口Line-5 mine-15

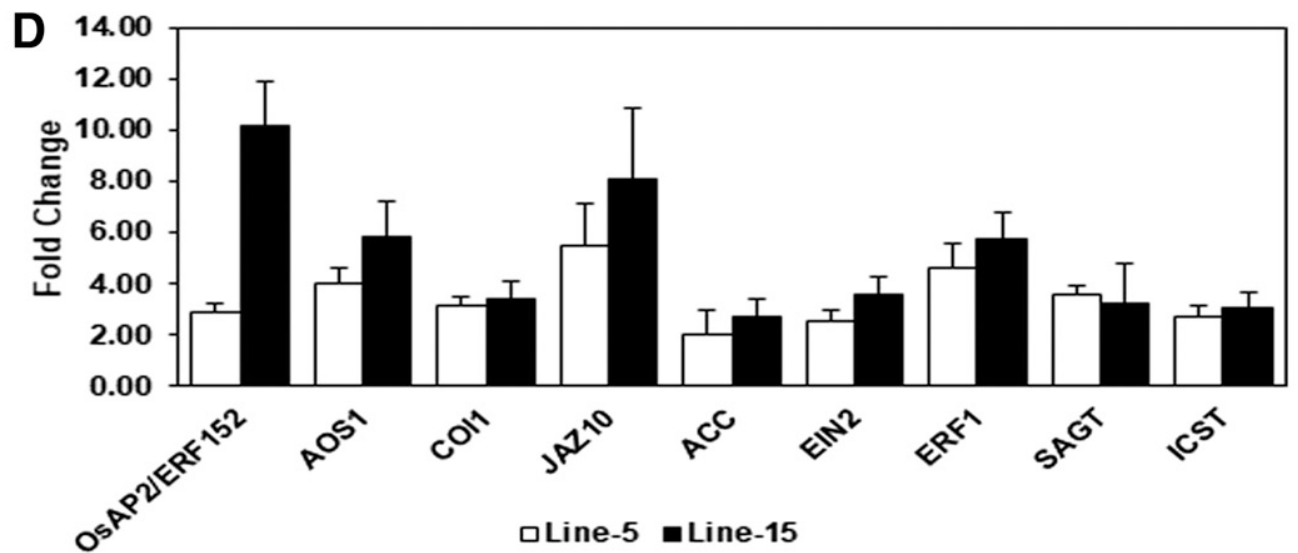

Fig. 2. Ectopic expression of OsAP2/ERF152 induces plant defense response. A, Leaves of 4-week-old of 17- $\beta$-estradiol-inducible XVE::OsAP2/ERF152 transgenic lines of $\mathrm{T}_{2}$ generations were infiltrated with either the XVE promoter inducer ( $20 \mu \mathrm{M}$ estradiol) or water (with dimethyl sulfoxide) (MQ) using a 1-ml needleless syringe. Leaves were harvested after $12 \mathrm{~h}$ and processed for aniline blue staining. Callose was observed as white spots under an epifluorescence microscope. The scale bar in the images represent $50 \mu \mathrm{m}$. B, Bars represent the mean value of number of callose deposits per field of view $(n=5)$. All of the data were analyzed using Student's $t$ test (* indicates significant difference with $P<0.05$ ). Leaves from 3- to 4-week-old XVE::OsAP2/ERF152 plants treated with either estradiol or water were harvested and processed for quantitative PCR analysis after $12 \mathrm{~h}$ of the treatment. WT $=$ wild type. C and $\mathbf{D}$, Expression of $O s A P 2 /$ ERF152 induced various defense response genes regulated by either salicylic acid (SA) or jasmonic acid/ethylene: Pathogenesis related-1 (PR1), PR2, PR3, Phytoalexin deficient-3 (PAD3), avrPphB susceptible-3 (PBS3), Allene oxide synthase-1 (AOS1), Coronatine-insensitive protein-1 (COI1), Jasmonate zim domain (JAZ10), 1-aminoacyclopropane 1-carboxylate synthase (ACC), Ethylene insensitive-2 (EIN2), Ethylene response factor-1 (ERF1), SA glycosyl transferase (SAGT), and Isochorismate synthase (ICST). The Arabidopsis UBQ5 gene was used as internal control for quantitative real-time PCR analysis. The graph represents the relative fold change $\left(2^{-} \Delta \Delta \mathrm{Ct}\right)$ calculated using expression values of induced over uninduced plants after $12 \mathrm{~h}$ of induction. The average from three replicates is plotted in the graph and error bars denote standard deviation. Similar results were obtained in three separate experiments with two independent XVE::OsAP2/ ERF152 transgenic lines. 


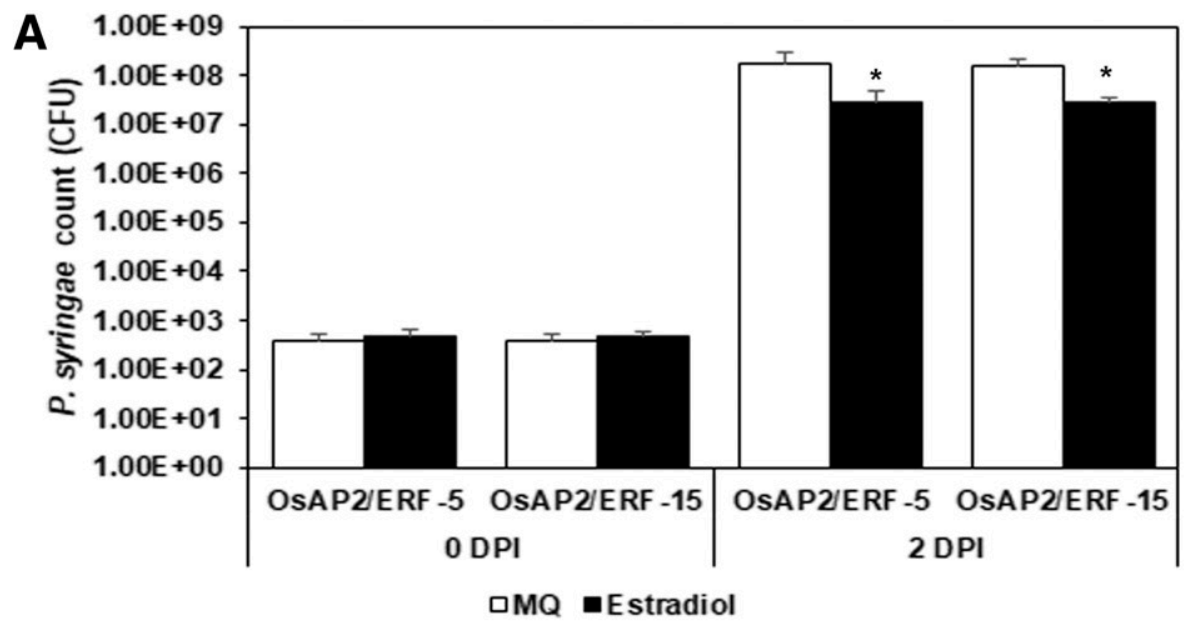

B

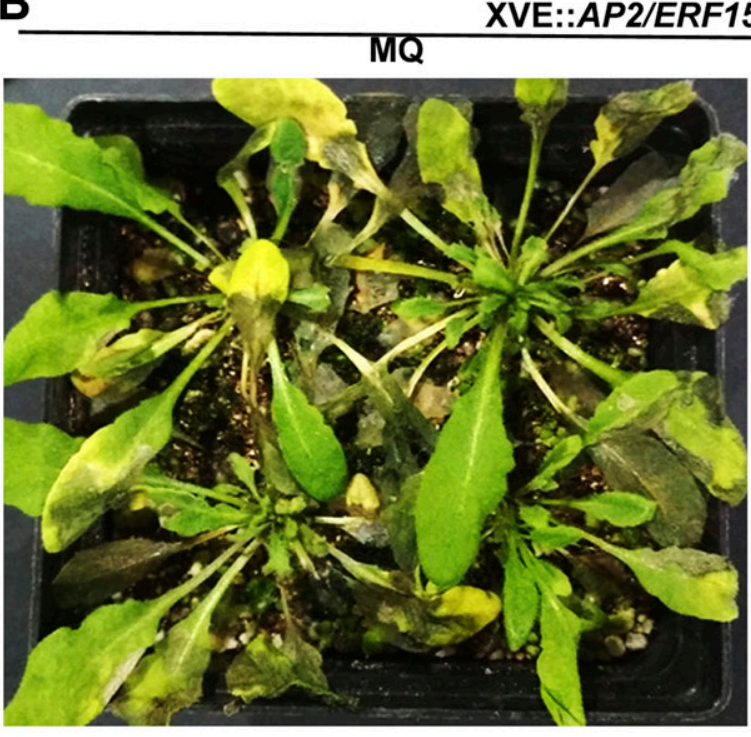

XVE::AP2/ERF152 + R.solani infection

C
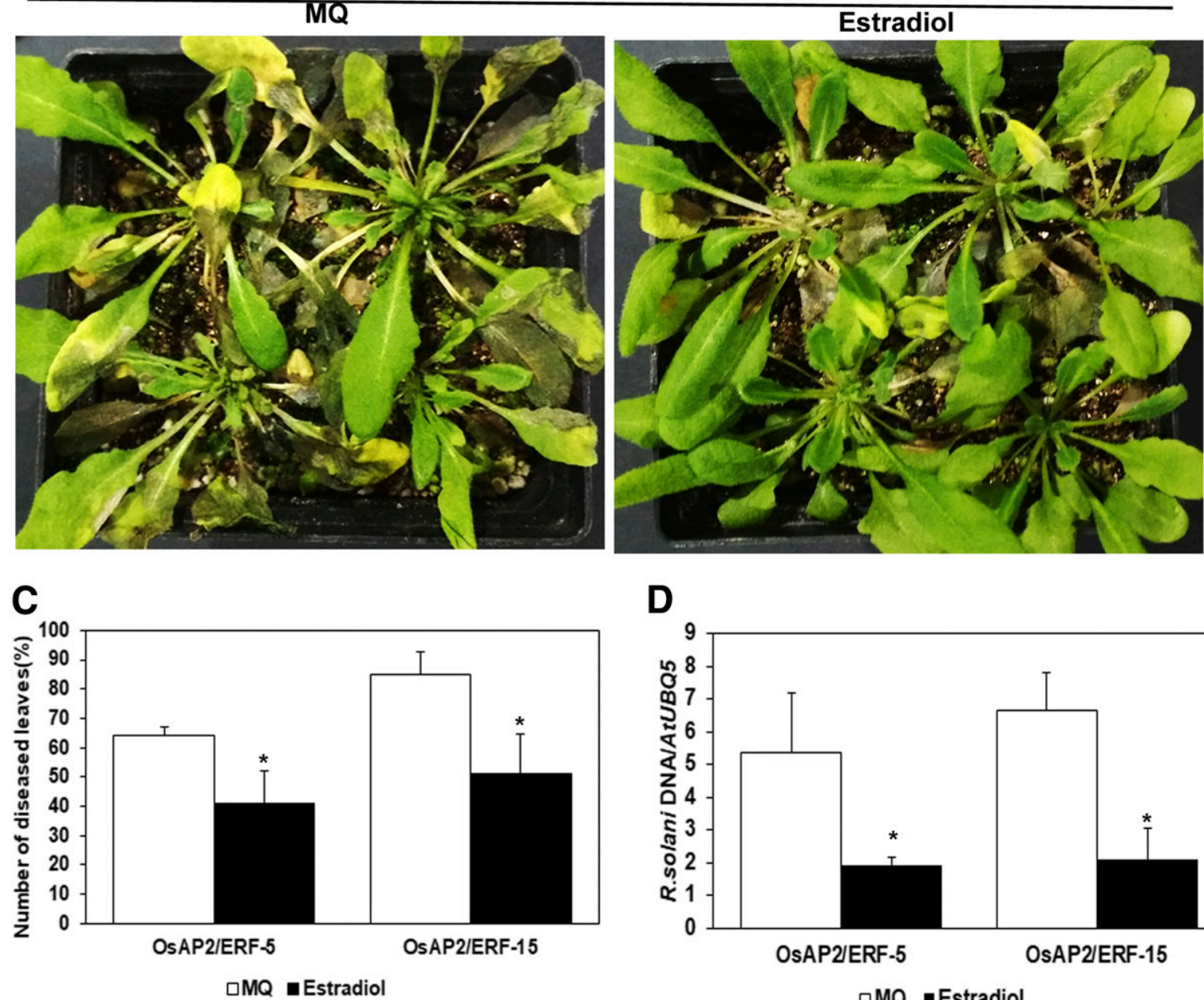

D

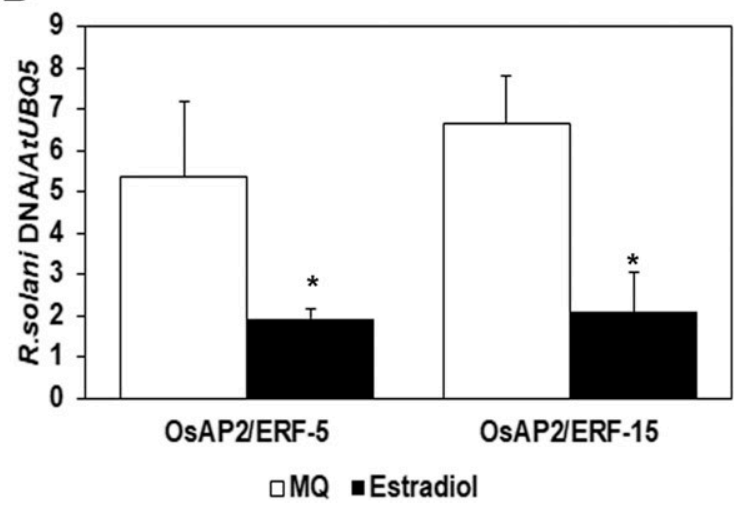

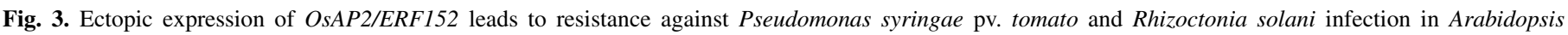

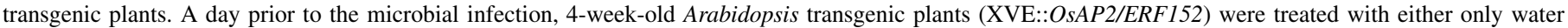

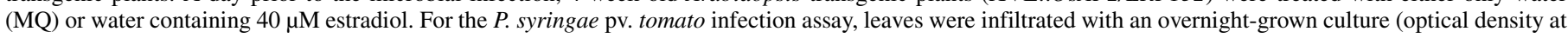

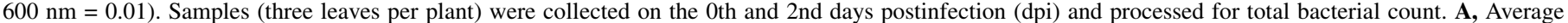

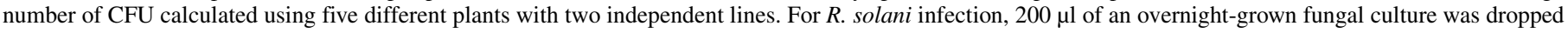

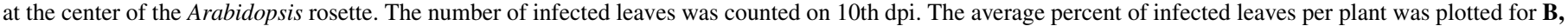

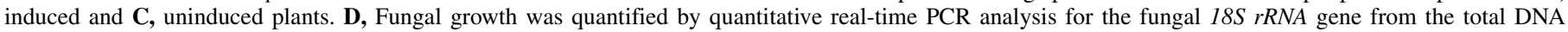

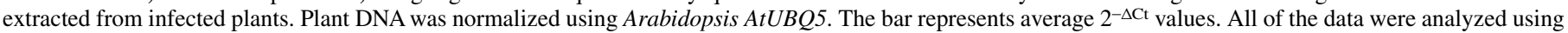

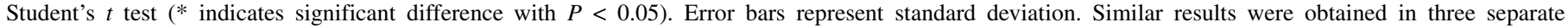
experiments with two independent XVE::OsAP2/ERF152 transgenic lines. 
Expression of $O s A P 2 / E R F 152$ provides resistance against $R$. solani infection in Arabidopsis. We then tested whether OsAP2/ERF 152 expression would lead to resistance to the necrotrophic pathogen $R$. solani. A suspension of an overnightgrown $R$. solani culture was inoculated at the center of the rosette of XVE::OsAP2/ERF152 transgenic plants pretreated with either estradiol or water. The number of leaves with disease symptoms was counted on the 10th day postinfection. In the transgenic plants that were pretreated with water (uninduced condition), $R$. solani infection caused either partially necrotic lesions or the entire leaf was dead (Fig. 3B). However, the younger leaves remained green and appeared to be healthy. The number of such diseased leaves was significantly lower in OsAP2/ERF152-expressing plants (induced condition) (Fig. 3C). Fungal growth was quantitated by checking the amplification of a fungal gene by qPCR. A significant $(P<0.05)$ reduction in fungal load was observed following induction of OsAP2/ERF152 expression (Fig. 3D), revealing that the expression of this rice transcription factor leads to resistance to infection caused by a necrotrophic pathogen in Arabidopsis. Treatment with estradiol alone did not affect $R$. solani disease progression in the wild-type Arabidopsis plants (Kachewar et al. 2019).

Constitutive expression of $O S A P 2 / E R F 152$ results in the reduction of seedling growth in Arabidopsis. Treatment with elicitors such as Flg22, oligogalacturonides, chitin, and cellobiose leads to growth inhibition in Arabidopsis seedlings (Denoux et al. 2008; Souza et al. 2017). The continuous activation of immune responses has adverse effects on plant growth (de Vries et al. 2017; Yang et al. 2012). Because $O s A P 2 / E R F 152$ induces plant immune responses such as callose deposition and expression of defense genes, we tested the effect of $O S A P 2 / E R F 152$ expression on plant growth. In order to study the effect of expression of OsAP2/ERF 152 on growth, the seedlings of XVE::OsAP2/ERF152 transgenic lines and wild-type plants were grown on MS agar medium in the presence and absence of estradiol. After 10 days of estradiol treatment, the fresh weight of individual seedlings was measured. Continuous induction of $O s A P 2 / E R F 152$ resulted in a significant reduction in the fresh weight. (Fig. 4). The rosette diameter of the seedlings remained unchanged while the root branching was significantly reduced. However, the inducer (estradiol) had no effect on the growth of wild-type seedlings. Similar results were obtained in two independently transformed transgenic lines across three independent experiments.

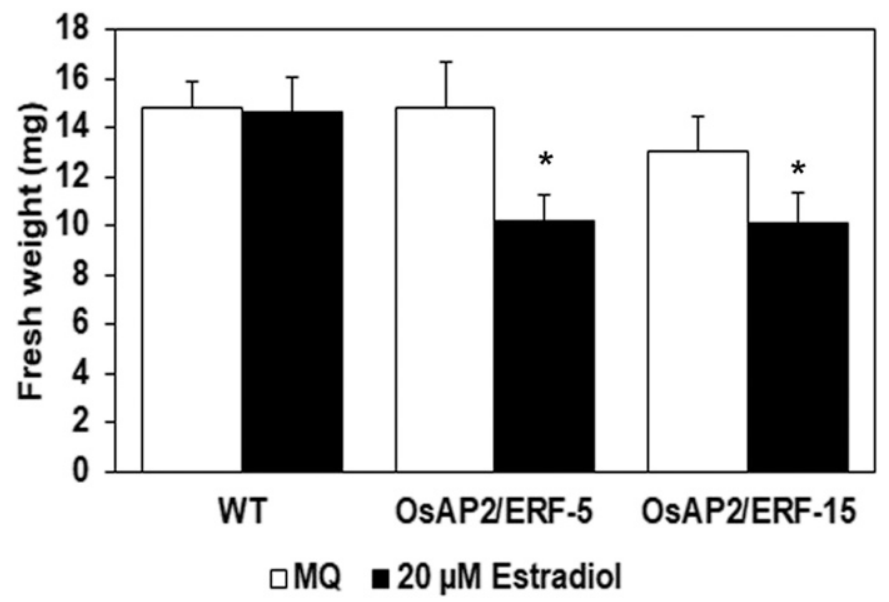

Fig. 4. Constitutive expression of $O s A P 2 / E R F 152$ has an inhibitory effect on plant growth. For assaying the effect on growth, seedlings of wild type (WT) and XVE::OsAP2/ERF152 were grown vertically on Murashige and Skoog agar medium in the absence (MQ) and presence of estradiol. On the 10th day, individual seedlings $(n=15)$ were weighed and the average fresh weight was plotted. The error bar represents standard deviation. Data were analyzed using Student's $t$ test ( $*$ indicates significant difference with $P<0.05$ ). Similar results were obtained in two separate experiments with two independent $\mathrm{XVE}:$ OsAP2/ERF152 transgenic lines.
Ectopic expression of $O s A P 2 / E R F 152$ activates MAPK signaling in Arabidopsis. MAPK signaling is induced in response to external as well as internal signals in plant cells. The activation of MAPKs is the early signaling event observed during PAMP-triggered immunity as well as DAMP-triggered immunity (Meng and Zhang 2013). Because MAPK signaling is an upstream signaling event, we tested its involvement in OsAP2/ERF152induced immunity. For this, phosphorylation of MPK3 and MPK6 were tested in 2-week-old wild-type and XVE::OsAP2/ERF152 transgenic lines. The seedlings were treated with estradiol for various durations $(0,10,15,30$, and $60 \mathrm{~min})$, followed by total protein extraction and Western blot analysis. The expression of OsAP2/ERF152 resulted in the transient activation of MPK3 and MPK6, which was initiated at 15 min and reached a maximum at 30 min post estradiol treatment (Fig. 5). The levels of phosphorylated MPK6 and -3 returned to the basal levels at $60 \mathrm{~min}$ in OsAP2/ ERF152-expressing seedlings. Tsuda et al. (2013) reported that estradiol treatment did not induce MPK3 and -6 activation in wildtype Arabidopsis. In the present study, a basal level of MPK3 and -6 was observed in wild-type seedlings which remained unchanged upon estradiol treatment (Fig. 5). We used Flg22 as a positive control for locating phosphorylated MPK3 and -6 (Supplementary Fig. S2). Similar results were obtained in two independent lines across two experiments.

\section{DISCUSSION}

The action of CWDEs releases cell wall degradation products which induce plant immune responses (Walton 1994). We have shown earlier that treatment with CWDEs induces a strong immune response potent enough to provide enhanced tolerance to bacterial infection in rice (Jha et al. 2007). Transcriptional reprogramming is a key step in the induction of an immune response and this process is generally executed by transcription factors. We initiated this study to characterize the $O s A P 2 / E R F 152$ transcription factor because it was highly upregulated in the transcriptome analysis performed upon treatment of rice leaves with ClsA (Jha et al. 2010). In rice, expression of $O s A P 2 / E R F 152$ is induced by a variety of CWDEs but not by Flg22 and LPS, suggesting that it may be specific for cell wall damage or CWDE-induced immune responses. Ectopic expression of $O s A P 2 / E R F 152$ leads to resistance against hemibiotrophic and necrotrophic infection in Arabidopsis. It is well reported that SA plays an active role in controlling the growth of a hemibiotrophic pathogen while the JA/ET-mediated plant defense response suppresses necrotrophic infection in Arabidopsis (Tsuda et al. 2009; Xin and He 2013). The resistance to $P$. syringae pv. tomato infection observed upon OsAP2/ERF152 expression could be due to the expression of SA-responsive defense genes. Independent studies show that the increased tolerance to $R$. solani infection in rice is associated with plant responses induced by either

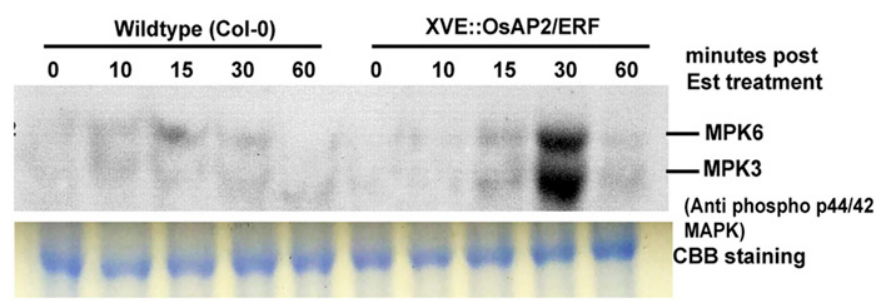

Fig. 5. Ectopic expression of $O s A P 2 / E R F 152$ leads to transient activation of MPK3 and MPK6 in Arabidopsis. For testing activation of MPK3 and -6, 2week-old seedlings ( $n=10$ to 15 ) of the wild type and XVE::OsAP2/ERF152 were treated with estradiol for different time points $(0,10,15,30$, and $60 \mathrm{~min})$ and processed for protein extraction followed by Western blotting using antiphospho p44/42 (Thr202/Tyr204) antibodies. MAPK = mitogen-activated protein kinase and $\mathrm{CBB}=$ Coomassie Brilliant Blue. Both of the above experiments were repeated twice in two independent transgenic lines. 
SA, JA, or ET (Kouzai et al. 2018). There are many studies reported where activation of a broad immune response is associated with the activation of both SA and JA response pathways. Treatment with Flg22, Bacillus cereus AR156 (a nonpathogenic rhizobacterium), or chitosan induce both the SA- and JA/ET-mediated immune response pathway in Arabidopsis (Denoux et al. 2008; Jia et al. 2018; Jiang et al. 2016). A similar result was reported in strawberry plants, where Colletotrichum acutatum (a fungal pathogen) partially activated the SA and JA/ET response pathways (Amil-Ruiz et al. 2016). Thus, the resistance to hemibiotrophic and necrotrophic infection in OsAP2/ERF152-expressing Arabidopsis lines may be due to the enhanced callose deposition and induction of various defense genes involved in the SA and JA/ET responses.

Induction of the DAMP-triggered immune response involves activation of MAPKs as one of the earliest events, and MPK3 and -6 are activated upon treatment with oligogalactourans and cellobiose in Arabidopsis plants (Meng and Zhang 2013; Souza et al. 2017). Ectopic expression of $O S A P 2 / E R F 152$ led to transient activation of MPK3 and MPK6 in Arabidopsis. Activation of MPK3 and MPK6 leads to induction of the ET response pathway in Arabidopsis. MPK3 plays an important role in activating the SA response while MPK6 partially induces expression of JAresponsive and biosynthetic genes (Meng and Zhang 2013). Previous studies show that activation of MPK3 and MPK6 leads to resistance to infection by $P$. syringae pv. tomato and a fungal pathogen, Botrytis cinerea, in Arabidopsis (Asai et al. 2002). Thus, we propose that the OsAP2/ERF152-mediated activation of a broad-range immune response may be due to the transient activation of MPK3 and -6 , which may lead to upregulation of SA- and JA/ET-responsive genes.

Overall, the present study demonstrates that ectopic expression of OsAP2/ERF 152 activates a broad range of immunity that leads to resistance to fungal and bacterial infection in Arabidopsis. This immunity involves activation of MPK3 and -6 , which may lead to enhanced expression of plant defense genes regulated by both SA and JA/ET hormones. Previous work by Jha et al. (2010) showed that transient expression of OsAP2/ERF152 leads to resistance to $X$. oryzae pv. oryzae infection in rice. The fact that overexpression of OsAP2/ERF152 leads to resistance to bacterial infection in rice and in Arabidopsis suggests that its overexpression may be helpful in providing tolerance to infection in different plants. However, a continuous expression of OsAP2/ERF152 leads to growth inhibition, suggesting that it must be used with a regulatable promoter; specifically, a defense-inducible promoter. Also, overexpression of OsAP2/ERF 152 provides a moderate level of tolerance, suggesting that it must be used in conjunction with other defense genes. Future work would be directed toward expressing OSAP2/ERF152 with a defense-inducible promoter in crop plants. Also, we would be looking to determine the exact role of OsAP2/ERF152 in CWDEinduced immune responses in rice through development of null mutants using genome editing techniques.

\section{ACKNOWLEDGMENTS}

We thank U. Grossniklaus (University of Zurich) and N.-H. Chua (Rockefeller University) for providing the vector pMDC7; and G. Jha (National Institute of Plant Genome Research), G. S. Laha (Indian Institute of Rice Research [IIRR]), and S. Chatterjee (Centre for DNA fingerprinting $\&$ Diagnostics [CDFD]) for providing microbial strains used in this study.

\section{LITERATURE CITED}

Amil-Ruiz, F., Garrido-Gala, J., Gadea, J., Blanco-Portales, R., Munoz-Merida, A., Trelles, O., de Los Santos, B., Arroyo, F. T., Aguado-Puig, A., Romero, F., Mercado, J. A., Pliego-Alfaro, F., Munoz-Blanco, J., and Caballero, J. L. 2016. Partial activation of SA- and JA-defensive pathways in strawberry upon Colletotrichum acutatum interaction. Front. Plant Sci. 7:1036.

Arvidsson, S., Kwasniewski, M., Riano-Pachon, D. M., and Mueller-Roeber, B. 2008. QuantPrime-A flexible tool for reliable high-throughput primer design for quantitative PCR. BMC Bioinf. 9:465.
Asai, T., Tena, G., Plotnikova, J., Willmann, M. R., Chiu, W. L., Gomez-Gomez, L., Boller, T., Ausubel, F. M., and Sheen, J. 2002. MAP kinase signalling cascade in Arabidopsis innate immunity. Nature 415:977-983.

Clough, S. J., and Bent, A. F. 1998. Floral dip: A simplified method for Agrobacterium-mediated transformation of Arabidopsis thaliana. Plant $\mathbf{J}$. 16:735-743.

Denoux, C., Galletti, R., Mammarella, N., Gopalan, S., Werck, D., De Lorenzo, G., Ferrari, S., Ausubel, F. M., and Dewdney, J. 2008. Activation of defense response pathways by OGs and Flg22 elicitors in Arabidopsis seedlings. Mol. Plant 1:423-445.

de Vries, J., Evers, J. B., and Poelman, E. H. 2017. Dynamic plant-plantherbivore interactions govern plant growth-defence integration. Trends Plant Sci. 22:329-337.

Jha, G., Patel, H. K., Dasgupta, M., Palaparthi, R., and Sonti, R. V. 2010. Transcriptional profiling of rice leaves undergoing a hypersensitive response like reaction induced by Xanthomonas oryzae pv. oryzae Cellulase. Rice 3:1-21.

Jha, G., Rajeshwari, R., and Sonti, R. V. 2007. Functional interplay between two Xanthomonas oryzae pv. oryzae secretion systems in modulating virulence on rice. Mol. Plant-Microbe Interact. 20:31-40.

Jia, X., Zeng, H., Wang, W., Zhang, F., and Yin, H. 2018. Chitosan oligosaccharide induces resistance to Pseudomonas syringae pv. tomato DC3000 in Arabidopsis thaliana by activating both salicylic acid- and jasmonic acidmediated pathways. Mol. Plant-Microbe Interact. 31:1271-1279.

Jiang, C.-H., Huang, Z.-Y., Xie, P., Gu, C., Li, K., Wang, D.-C., Yu, Y.-Y., Fan, Z.-H., Wang, C.-J., Wang, Y.-P., Guo, Y.-H., and Guo, J.-H. 2016. Transcription factors WRKY70 and WRKY11 served as regulators in rhizobacterium Bacillus cereus AR156-induced systemic resistance to Pseudomonas syringae pv. tomato DC3000 in Arabidopsis. J. Exp. Bot. 67:157-174.

Kachewar, N. R., Gupta, V., Ranjan, A., Patel, H. K., and Sonti, R. V. 2019. Overexpression of OsPUB41, a rice E3 ubiquitin ligase induced by cell wall degrading enzymes, enhances immune responses in rice and Arabidopsis. BMC Plant Biol. 19:530.

Kouzai, Y., Kimura, M., Watanabe, M., Kusunoki, K., Osaka, D., Suzuki, T., Matsui, H., Yamamoto, M., Ichinose, Y., Toyoda, K., Matsuura, T., Mori, I. C., Hirayama, T., Minami, E., Nishizawa, Y., Inoue, K., Onda, Y., Mochida, K., and Noutoshi, Y. 2018. Salicylic acid-dependent immunity contributes to resistance against Rhizoctonia solani, a necrotrophic fungal agent of sheath blight, in rice and Brachypodium distachyon. New Phytol. 217:771-783.

Licausi, F., Ohme-Takagi, M., and Perata, P. 2013. APETALA2/ethylene responsive factor (AP2/ERF) transcription factors: Mediators of stress responses and developmental programs. New Phytol. 199:639-649.

Livak, K. J., and Schmittgen, T. D. 2001. Analysis of relative gene expression data using real-time quantitative PCR and the $2^{-\Delta \Delta C T}$ method. Methods 25 : $402-408$

Meng, X., and Zhang, S. 2013. MAPK cascades in plant disease resistance signaling. Annu. Rev. Phytopathol. 51:245-266.

Millet, Y. A., Danna, C. H., Clay, N. K., Songnuan, W., Simon, M. D., Werck-Reichhart, D., and Ausubel, F. M. 2010. Innate immune responses activated in Arabidopsis roots by microbe-associated molecular patterns. Plant Cell 22:973-990.

Murashige, T., and Skoog, F. 1962. A revised medium for rapid growth and bio assays with tobacco tissue cultures. Physiol. Plant. 15:473-497.

Okubara, P. A., Schroeder, K. L., and Paulitz, T. C. 2008. Identification and quantification of Rhizoctonia solani and $R$. oryzae using real-time polymerase chain reaction. Phytopathology 98:837-847.

Pillai, S. E., Kumar, C., Patel, H. K., and Sonti, R. V. 2018. Overexpression of a cell wall damage induced transcription factor, OsWRKY42, leads to enhanced callose deposition and tolerance to salt stress but does not enhance tolerance to bacterial infection. BMC Plant Biol. 18:177.

Ranjan, A., Vadassery, J., Patel, H. K., Pandey, A., Palaparthi, R., Mithofer, A., and Sonti, R. V. 2015. Upregulation of jasmonate biosynthesis and jasmonate-responsive genes in rice leaves in response to a bacterial pathogen mimic. Funct. Integr. Genomics 15:363-373.

Sharoni, A. M., Nuruzzaman, M., Satoh, K., Shimizu, T., Kondoh, H., Sasaya, T., Choi, I.-R., Omura, T., and Kikuchi, S. 2011. Gene structures, classification and expression models of the AP2/EREBP transcription factor family in rice. Plant Cell Physiol. 52:344-360.

Souza, C. A., Li, S., Lin, A. Z., Boutrot, F., Grossmann, G., Zipfel, C., and Somerville, S. C. 2017. Cellulose-derived oligomers act as damageassociated molecular patterns and trigger defense-like responses. Plant Physiol. 173:2383-2398.

Starkey, M., and Rahme, L. G. 2009. Modeling Pseudomonas aeruginosa pathogenesis in plant hosts. Nat. Protoc. 4:117-124.

Tsuda, K., Mine, A., Bethke, G., Igarashi, D., Botanga, C. J., Tsuda, Y., Glazebrook, J., Sato, M., and Katagiri, F. 2013. Dual regulation of gene 
expression mediated by extended MAPK activation and salicylic acid contributes to robust innate immunity in Arabidopsis thaliana. PLoS Genet. 9:e1004015.

Tsuda, K., Sato, M., Stoddard, T., Glazebrook, J., and Katagiri, F. 2009. Network properties of robust immunity in plants. PLoS Genet. 5:e1000772.

Walton, J. D. 1994. Deconstructing the cell-wall. Plant Physiol. 104: 1113-1118.

Xin, X.-F., and He, S. Y. 2013. Pseudomonas syringae pv. tomato DC3000: A model pathogen for probing disease susceptibility and hormone signaling in plants. Annu. Rev. Phytopathol. 51:473-498.
Xu, K., Huang, X., Wu, M., Wang, Y., Chang, Y., Liu, K., Zhang, J., Zhang, Y., Zhang, F., Yi, L., Li, T., Wang, R., Tan, G., and Li, C. 2014. A rapid, highly efficient and economical method of Agrobacterium-mediated in planta transient transformation in living onion epidermis. PLoS One 9: e83556.

Yang, D. L., Yao, J., Mei, C. S., Tong, X. H., Zeng, L. J., Li, Q., Xiao, L. T., Sun, T. P., Li, J., Deng, X. W., Lee, C. M., Thomashow, M. F., Yang, Y., He, Z., and He, S. Y. 2012. Plant hormone jasmonate prioritizes defense over growth by interfering with gibberellin signaling cascade. Proc. Natl. Acad. Sci. U.S.A. 109:E1192-E1200. 